\title{
Effect of aldosterone on the amplification of oncolytic vaccinia virus in human cancer lines
}

\author{
Hyun Ju Lee ${ }^{1^{*}}$, Jasung Rho ${ }^{1^{*}}$, Shao Ran Gui ${ }^{2}$, Mi Kyung Kim² , Yu Kyoung Lee ${ }^{2}$, Yeon Sook Lee ${ }^{2}$, \\ Jeong Eun $\mathrm{Kim}^{1}$, Euna $\mathrm{Cho}^{2}$, Mong $\mathrm{Cho}^{3}$, and Tae-Ho Hwang ${ }^{1,4}$ \\ ${ }^{1}$ Department of Pharmacology, Pusan National University School of Korean Medicine; ${ }^{2}$ Department of \\ Pharmacology, Pusan National University School of Medicine; ${ }^{3}$ Department of Gastroenterology, Pusan National \\ University College of Medicine, Busan; ${ }^{4}$ Research Center for Hepatic and Biliary Cancer Center, \\ Pusan National University Yangsan Hospital, Pusan National University College of Medicine, Yangsan, Korea
}

\begin{abstract}
Background/Aims: JX-594 is an oncolytic virus derived from the Wyeth vaccinia strain that causes replication-dependent cytolysis and antitumor immunity. Starting with a cross-examination of clinical-trial samples from advanced hepatocellular carcinoma patients having high levels of aldosterone and virus amplification in JX-594 treatment, we investigated the association between virus amplification and aldosterone in human cancer cell lines. Methods: Cell proliferation was determined by a cell-counting-kit-based colorimetric assay, and vaccinia virus quantitation was performed by quantitative polymerase chain reaction (qPCR) and a viral plaque assay. Also, the intracellular $\mathrm{pH}$ was measured using a pH-sensitive dye. Results: Simultaneous treatment with JX-594 and aldosterone significantly increased viral replication in A2780, PC-3, and HepG2 cell lines, but not in U2OS cell lines. Furthermore, the aldosterone treatment time altered the JX-594 replication according to the cell line. The JX-594 replication peaked after 48 and 24 hours of treatment in PC-3 and HepG2 cells, respectively. qPCR showed that JX-594 entry across the plasma membrane was increased, however, the changes are not significant by the treatment. This was inhibited by treatment with spironolactone (an aldosterone-receptor inhibitor). JX-594 entry was significantly decreased by treatment with EIPA [5-(N-ethyl-N-isopropyl)amiloride; a Na ${ }^{+} / \mathrm{H}^{+}$-exchange inhibitor], but aldosterone significantly restored JX-594 entry even in the presence of EIPA. Intracellular alkalization was observed after aldosterone treatment but was acidified by EIPA treatment. Conclusions: Aldosterone stimulates JX-594 amplification via increased virus entry by affecting the $\mathrm{H}^{+}$gradient. (Korean $\mathrm{J}$ Hepatol 2011;17:213-219)
\end{abstract}

Keywords: Aldosterone; Vaccinia virus; Mineralocorticoid receptor; pH; Virus entry

\section{INTRODUCTION}

JX-594 is a targeted, GM-CSF expressing Wyeth strain derived oncolytic virus designed to selectively replicate in and destroy cancer cells through virus replication-dependent cytolysis and induction of anti-tumoral immunity. ${ }^{1,2}$ JX-594 replication in cancer patients seem to be associated with genetic background of cancer cells and patient's condition as well as the administered viral dose. As viral replication in oncolytic virus therapy may be essential for therapeutic success, the favorable condition for
JX-594 replication should be illuminated. In phase 1 hepatoma clinical trial of JX-594, viral replication of JX-594 was noted in most patients, ${ }^{3}$ remarkably more in cancer patients with severe ascites and/or peripheral edema. It is assumed that aldosterone level is increased in the malignancy patients with ascites which is the abnormal accumulation of fluid within the peritoneal cavity as a consequence of advance cancer. Sequestration of fluid within the abdomen leads to additional fluid retention by the kidney due to stimulatory effect on blood pressure hormones, notably aldosterone which can cause peripheral edema.

Received June 20, 2011; Revised August 16, 2011; Accepted August 29, 2011

Abbreviations: BCECF-AM, 2-,7-bis-(2-carboxyethyl)-5-(and-6)-carboxyfluorescein acetoxymethyl ester; EIPA, 5-(N-Ethyl-N-isopropyl) amiloride; GM-CSF, granulocyte macrophage-colony stimulating factor; MOI, multiplicity of infection; MR, mineral corticoid receptor Comesponding author: Mong Cho

Department of Gastroenterology, Pusan National University Yangsan Hospital, Beomeo-ri, Mulgeum-eup, Yangsan 626-770, Korea Tel. +82-55-360-2350, Fax. +82-55-360-2150, E-mail; mcho@pusan.ac.kr

* These authors contributed equally to this work. 
Aldosterone is a type of mineralcorticoid hormone to control the balance of water and salts in the kidney by keeping sodium in and releasing potassium from the body. Aldosterone receptor, mineral corticoid receptor (MR), belongs to the nuclear receptor family where the ligand diffuses into cells, which interacts with the receptor and results in a signal transduction affecting specific gene expression in the nucleus. Pathophysiological function of aldosterone is well known as a cardiovascular function, but gene expression of MR is ubiquitous in most normal organs including cancer cells with higher levels in the cortex, amygdala and the pituitary gland (5-20 folds of average), cardiomyocytes, uterus and lymphocytes (3-5 folds of average) shown in Norvatis Research Foundation based BioGPS site. ${ }^{4}$ This means that a variety of physiological functions other than fluid regulation by aldosterone is possible. Accordingly, we addressed the effect of aldosterone in JX-594 amplification in vitro in this study.

\section{MATERIALS AND METHODS}

\section{Chemicals}

Aldosterone, spirinolactone and 5-(N-Ethyl-N-isopropyl) amiloride (EIPA) were purchased from Sigma Aldrich. 2-, 7-bis-(2-carboxyethyl)-5-(and-6)-carboxyfluorescein acetoxymethyl ester (BCECF-AM) from Molecular Probe (Eugene, Ore., USA).

\section{Viruses and cell lines}

JX-594 was constructed from a Wyeth strain vaccinia virus (vv) as described, ${ }^{5}$ and JX-963 was constructed from a Western Reserve vaccinia virus as described. ${ }^{6}$ Both wild type Wyeth strain vV and WR strain vv were purchased from ATCC. Human hepatocellular carcinoma cell lines (SNU-449 and SNU-475) were obtained from Korea Cell Bank (Seoul, Korea) and all other cell lines used were obtained from ATCC.

\section{Measurement of cell viability}

Cell Counting Kit-8 (CCK-8, Dojindo Molecular Technologies, Inc., Japan) was used in accordance with a company provided manual to determine cell growth. In brief, the cells were plated in 96-well plates 1 day before treatment; pre-incubated cells with aldosterone $(48 \mathrm{~h})$ or vehicle were washed with PBS, and then 10 $\mu \mathrm{l}$ of CCK- 8 was added to the cells and plated for 2-4 hours in a humidified incubator (at $37^{\circ} \mathrm{C}, 5 \% \mathrm{CO}_{2}$ ), after which their absorbance at $450 \mathrm{~nm}$ were measured using ELISA reader
(ELx808 ${ }^{\mathrm{TM}}$ Absorbance Microplate Reader, Biotech, Winooski, VT, USA). The percentage of viable cells was calculated as the ratio of viable cells to total cells absorbance measurements $\times 100$.

\section{In vitro replication and cytopathic effect}

Cell lines were seeded into 6 -well plates at $4 \times 10^{5}$ cells/well and left overnight. Aldosterone and/or MR inhibitor, spirinolactone, were pretreated for different hours $(0,2,4,6,8,10)$ before virus infection. Virus was then added at an MOI of 1.0 PFU/cell with or without aldosterone and/or spironolactone and allowed to infect for 2 hours. At the end of the infection, the media was changed and plates incubated for 2 hours, 24 hours or 48 hours; the cells were then scraped into the media and collected. Cells were lysed by 3 rounds of freezing and thawing followed by sonication before serial dilutions of the crude viral lysate was added to A2780 cells to titer the virus by plaque assay. All experiments were run in triplicate. As vv replication was highest after pretreatment of aldosterone for 8-10 hour, all study otherwise indicated were conducted on this condition.

\section{Measurement of plasma membrane influx of vaccinia virus}

As vv replication was highest in PC-3 cell line after 48 hours incubation, qPCR quantitation in PC-3 cell line was used to determine vv influx. $4 \times 10^{5}$ PC-3 cell/well were seeded in 6 well plate $4 \times 10^{5}$ cells/well and incubated overnight. Before virus infection, cells were washed with cold PBS and cooled to $4^{\circ} \mathrm{C}$ and incubated for $1 \mathrm{~h}$ at $4^{\circ} \mathrm{C}$ with indicated (mock, 0.001-10 MOI) amounts of virus to proceed virus adsorption. ${ }^{7}$ Cells are washed with cold PBS twice to remove unbound virions and further incubate at $37^{\circ} \mathrm{C}$ for $30 \mathrm{~min}$ with prewarmed complete media. Total DNA was isolated using QIAamp DNA Blood mini Kit (Qiagen, Hilden, Germany) and quantified using NanoDrop ND-1000 spectrophotometer (PeqLab, Erlangen, Germany). The copy numbers of genome were quantified using primer set specific for 9EL gene (E9L-F1880 5'-GAA CAT TTT TGG CAG AGA GAG CC-3' E9L-R2057 5'-CAA CTC TTA GCC GAA GCG TAT GAG-3' E9L-p1924S-MGB 6'FAM-CAG GCT ACC AGT TCA A-MGBNFQ-3') and 10 ng, 100 ng of DNA template using ABI 7300 realtime PCR machine (Applied Biosystems, Warrington, UK). To test the effect of aldosterone or $\mathrm{Na}^{+} / \mathrm{H}^{+}$inhibitor, EIPA, on virus entry, cells were preincubated for 2 hours at $37^{\circ} \mathrm{C}$ with aldosterone $(100 \mathrm{nM})$ or spirinolactone $(1 \mu \mathrm{M})$ which were diluted in serum free media. 


\section{Measurement of intracellular $\mathrm{pH}(\mathrm{pH} i)$}

Intracellular $\mathrm{pH}$ was determined using the $\mathrm{pH}$-sensitive dye 2-,7-bis-(2-carboxyethyl)-5-(and-6)-carboxyfluorescein acetoxymethyl ester (BCECF-AM) as described. ${ }^{8}$ PC-3 cells were prepared for $\mathrm{pH} i$ as follows. $5 \times 10^{4}$ cells/well were seeded in 24 well plates and incubated overnight. After preincubation with $100 \mu \mathrm{M}$ aldosterone or serum free for $2 \mathrm{hr}$ at $37^{\circ} \mathrm{C}$ cells were loaded with $2 \mu \mathrm{M}$ BCECF-AM (Molecular Probes) for 30 minutes at $37^{\circ} \mathrm{C}$ diluted in HBSS buffer. After washing twice with HBSS and further incubation with vv for 30 minutes, relative fluorescence intensity of treatment groups were measured with Infinite F500 Multi-functional Microplate Reader (Tecan Trading AG, Mannedorf, Switzerland).

\section{Statistical analysis}

Values are given as mean $\pm \mathrm{SD}$ (standard deviation). Statistical evaluations of data were analyzed using student t test. $P$-value of less than 0.05 was defined as statistically significant.

\section{RESULTS}

Among patients with significant malignant ascites who were enrolled into phase 1 trial, ${ }^{3}$ archival serum (supine position) was available in one patient (\#303 patient) to measure aldosterone/ cortisol level (Table 1). Cortisol level was stable roughly within the normal range $(5-25 \mu \mathrm{g} / \mathrm{dL})$, but aldosterone level was maintained at around 10 folds higher than the normal range (7.5-150 ng/L at supine position) before JX-594 injection. qPCR based whole blood viral particles after treatment of JX-594 were determined at concomitant time-series. It showed significant level on day 5 and further increase on day 8 exhibiting remarkable JX-594 replication in this patient. This contrasts with viral clearance on day 8 for most patients even when patients show virus replication from day 3 (data not shown).

In pilot animal toxicology study, JX-963 with a dosage 1,000 folds higher than possible therapeutic dose $\left(1.3 \times 10^{7} \mathrm{pfu} / \mathrm{kg}\right)$, was intravenously injected into normal rabbit, and cortisol level and aldosterone level were increased by 3-4 folds and by 4-20 folds, respectively (Table 2), which may have resulted from acute inflammatory change of histological finding in adrenal gland (data not shown). In case of \#303 patient, the basal level of

Table 1. Changes in serum cortisol, aldosterone, and JX-594 viral particles in a patient with hepatocellular carcinoma after JX-594 injection

\begin{tabular}{|c|c|c|c|}
\hline Days post JX-594 & Cortisol (mg/dL) & Aldosterone (ng/L) & JX-594 (vp/mg DNA) \\
\hline D0 & 31.9 & 1551.2 & 0 \\
\hline D1 (15 min post JX-594) & 21.8 & 841.7 & 2,189 \\
\hline D5 & 28.5 & 937.8 & 6,137 \\
\hline D8 & 28.5 & 1482.2 & 11,891 \\
\hline
\end{tabular}

This patient was enrolled into JX-594 Phase 1 hepatoma trial.

Table 2. Changes in serum cortisol, aldosterone, and vaccina virus particles in normal rabbits ( $\mathrm{n}=4)$ after JX-594 injection

\begin{tabular}{lcccc}
\hline Dose of vv & Days post vv & Cortisol $(\mathrm{mg} / \mathrm{dL})$ & Aldosterone $(\mathrm{ng} / \mathrm{L})$ & vv viral particles $(\mathrm{vp} / \mathrm{mg}$ DNA) \\
\hline $5 \times 10^{7} \mathrm{pfu}$ & D0 & $0.7 \pm 0.2$ & $109.8 \pm 14$ & 0 \\
& D3 & $0.9 \pm 0.3$ & $165.3 \pm 45$ & $88.6 \pm 32$ \\
D5 & $1.0 \pm 0.5$ & $66.3 \pm 36$ & $88.7 \pm 22$ & 0 \\
$5 \times 10^{10} \mathrm{pfu}$ & D8 & $0.6 \pm 0.5$ & $496.3 \pm 102^{*}$ & 0 \\
& D0 & $0.8 \pm 0.4$ & $311.2 \pm 88^{*}$ & 32 \\
& D1 & $1.0 \pm 0.3$ & 9257 \\
\hline
\end{tabular}

${ }^{*} P<0.05$ (versus D0 level). 
aldosterone was high which may have affected JX-594 amplification. The effect of high dose JX-963 in adrenal gland stimulation needs further experimentation but was not further studied since it was not the primary focus of this study. In this study, $5 \times 10^{7}$ pfu injection showed no change in aldosterone, cortisol level, histological finding, and in JX-963 amplification.

Thus, direct effect of aldosterone on JX-594 amplification in human cell lines were determined (Fig. 1). As A2780 cancer cell line has been used for viral titering during Phase 1 and Phase 2 JX-594 study, A2780 cell line was initially chosen to determine the aldosterone effect on JX-594 amplification. Simultaneous treatment of JX-594 and aldosterone (10 nM) significantly increased viral replication in A2780 (human ovarian cancer cell line), which was completely blocked by co-treatment with MR inhibitor, spirinolactone (Fig. 1A). However, treatment of cortisol alone did not affect viral replication (data not shown). As aldosterone effect may be induced on both genomic and/or nongenomic level, ${ }^{9}$ we determined whether pretreatment time of aldosterone (10 nM) could influence viral replication (Fig. 1B). While there was significant increase in JX-594 replication after 0 (simultaneous treatment) to 6 hours pre-treatment of aldosterone, further increase after 8 or 10 hour treatment was observed.

Subsequently, it was determined whether the stimulation of JX-594 amplification by aldosterone $(10 \mathrm{nM})$ can vary depending on human cancer cell lines. Fig. 1C shows that there were minor to major differences in JX-594 replications depending on cell types. PC-3 cell showed the highest JX-594 replication after 48 hour treatment but HepG2 cell showed its peak replication after 24 hour treatment. Even with different patterns of aldosterone effect in different cell lines, the overall stimulatory effect of aldosterone in JX-594 amplification in PC3 cell and HepG2 cells but not in U2OS cell was observed.

As aldosterone has been reported to proliferate some types of cells such as heart cell, ${ }^{10}$ peripheral blood monocytes ${ }^{11}$ or human uterine leiomyoma, ${ }^{12}$ it was determined that the stimulation of JX-594 amplification may be via cell proliferation by aldosterone.
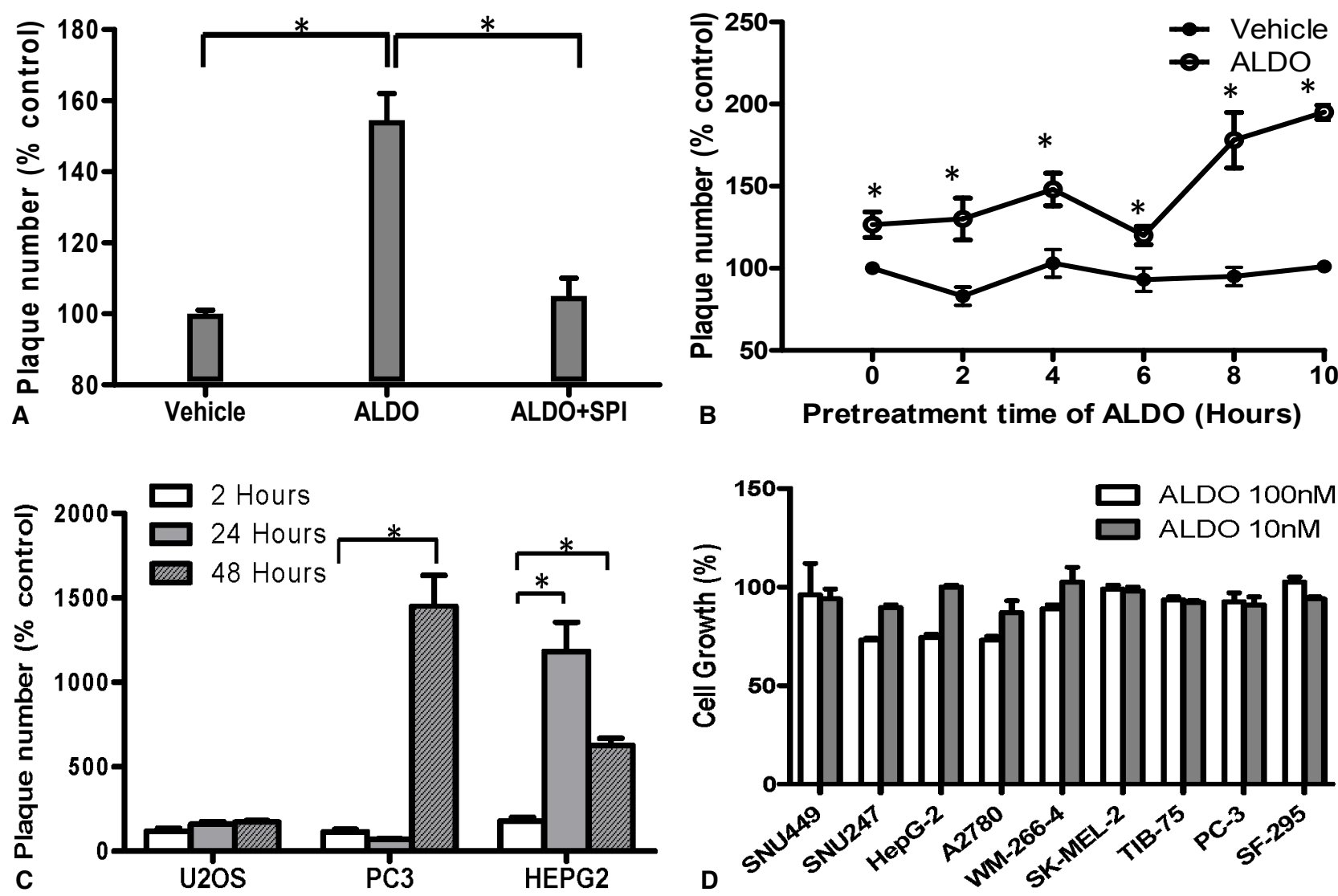

Figure 1. Effects of aldosterone (ALDO), spironolactone (SPI), and JX-594 on cancer cell lines (n=4). (A) Effects of treatment with ALDO (100 nmol) and/or SPI (100 nmol), and JX-594 (100 pfu) infection for 2 hours on A2780 cells ( $\mathrm{n}=6$ for each group). (B) Effects of pretreatment with ALDO (100 nmol) in JX-594 infection for 2, 4, 6, 8, and 10 hours ( $\mathrm{n}=3$ for each group) on virus replication. ${ }^{*} P<0.05$ (ALDO vs. vehicle at each time point). (C) U20S, PC-3, and HepG2 cell lines infected with JX-594 for 2, 24 , and 48 hours after 10 hours of treatment with ALDO (n=3). (D) Effects of ALDO at 10 and $100 \mathrm{nM}$ on tumor cell growth $(\mathrm{n}=2)$ ). ${ }^{*} P<0.05$. 

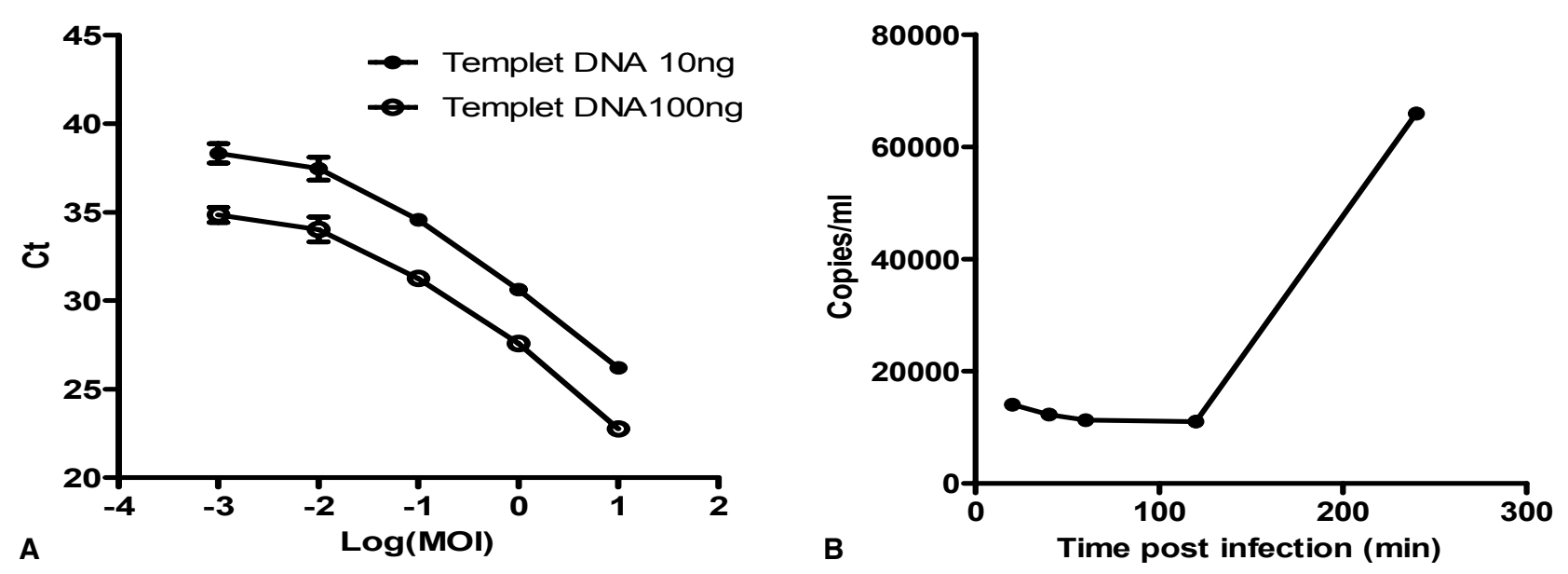

Figure 2. Results of quantitative polymerase chain reaction (qPCR) assay of intracellular vaccinia virus in PC-3 cells ( $\mathrm{n}=3$ ). (A) Linearity of $\mathrm{Ct}$ (cycle threshold) from qPCR using different dosage of templet. (B) Time-dependent viral amplification in PC-3. MOI, multiplicity of infection.

Fig. 1D indicates aldosterone $(10 \mathrm{nM})$ does not affect cell growth significantly in cancer cell lines we tested while high dose of aldosterone (100 $\mathrm{nM})$ may slightly inhibit cell proliferation in some cell lines. These results suggest no association of viral replication with aldosterone mediated cell growth.

As vaccinia virus enters cell in an extracellular $\mathrm{H}^{+}$dependent fashion ${ }^{7,13}$ and aldosterone can cause a change in cytosolic $\mathrm{pH}$ in specific cell lines, ${ }^{14,15}$ we addressed the possibility that change in hydrogen gradient across the plasma membrane can be involved in stimulation of JX-594 amplification in this study. For this purpose, we developed an assay for JX-594 entry into plasma membrane (Fig. 2). In this assay, qPCR based quantification of JX-594 entry after its treatment into cultured cells showed linearity plot of $\mathrm{Ct}$ and treated viral doses of 1-0.01 MOI (Fig. 2A). JX-594 copy number was stable until 2 hours post treatment, but significant increase at 4 hour post JX-594 treatment suggesting no viral replication at least for 2 hours post JX-594 treatment (Fig. 2B). Therefore we chose the condition of 30 minutes treatment after administration of 1 MOI JX-594 to measure viral entry but not replication. Quality controlled qPCR assay for clinical samples ${ }^{3}$ was used in this study. Fig. 3 shows that aldosterone slightly but not statistically increased viral entry. However, spirinolactone alone inhibited basal level of viral entry suggesting that basal MR activity may be involved in JX-594 entry. Simultaneous treatment of aldosterone and spirinolactone inhibited aldosterone stimulated viral entry but not as much as spirinolactone alone suggesting competition between aldosterone and spirinolactone. $\mathrm{Na}^{+} / \mathrm{H}^{+}$exchanger inhibitor, EIPA, significantly decreased basal level of JX-594 entry but this was

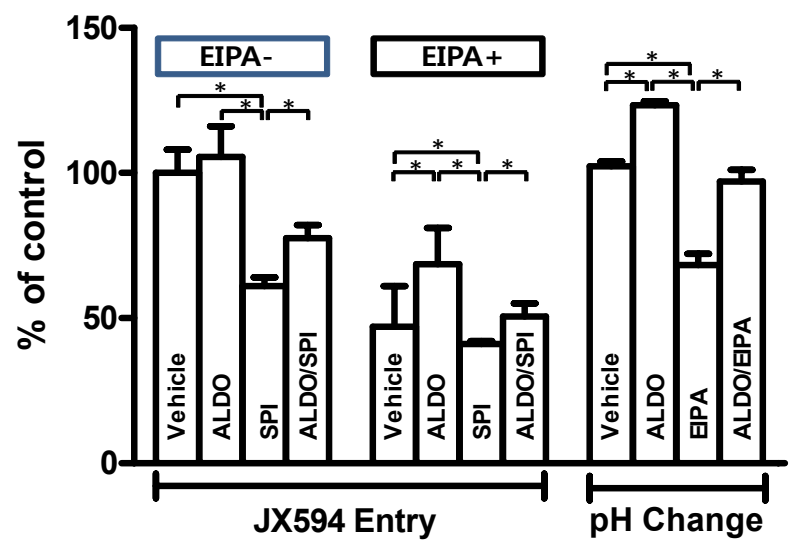

Figure 3. Results of qPCR quantification assay of intracellular vaccinia virus in $\mathrm{PC}-3$ cells, and $\mathrm{pH}$ changes observed after treatment with/without ALDO alone, or ALDO with SPI in the presence or absence of EIPA. $\mathrm{n}=3$ for each group, ${ }^{*} P<0.05$. EIPA, 5-(N-ethyl-N-isopropyl) amiloride.

slightly recovered by aldosterone treatment. This suggests that competition between aldosterone and spirinolactone is present also in the presence of EIPA. Then, we measured intracellular $\mathrm{pH}$ after aldosterone treatment in PC-3 cell. Aldosterone stimulated intracellular alkalization which was blocked by EIPA. Simultaneous treatment of aldosterone and EIPA recovered EIPA mediated cytosolic acidification but not to as much as aldosterone alone mediated alkalization suggesting competition between aldosterone and EIPA. Spirinoractone treated with/without aldosterone showed no significant effect in $\mathrm{pH}$ level which possibly suggests study limitations in interpreting this assay (data not shown). 


\section{DISCUSSION}

Viral replication in oncolytic viral therapeutics is a doubleedged sword. Efficient replication of oncolytic virus is essential for successful treatment. In contrast, in immune deficient patients, uncontrollable viral replication can cause serious clinical conditions. Therefore, pharmacological modulation of oncolytic virus replication may be the key to maximize the therapeutic window of oncolytic viruses.

Aldosterone level may be increased in terminal phase of tumor malignancy patients. Accordingly any cancer drug which potentially interacts with aldosterone should be used cautiously. As shown in Fig. 1B, treatment time of aldosterone may have different effect on $\mathrm{vV}$ replication suggesting aldosterone effect on a genomic level. This was further confirmed in Fig. 1C showing $\log$ scale increase of viral replication after $24 \mathrm{hrs}$ and $48 \mathrm{hrs}$ post infection. We speculate non-genomic level to be involved in aldosterone as well, and this can be seen in transient treatment of aldosterone affecting $\mathrm{pH}$ and virus entry (Fig. 3). Overall, the results of this study show that aldosterone can increase vaccinia virus entrance in vitro which may be responsible for increased viral amplification. The reason for minimum increase of viral entry by aldosterone determined by qPCR in this study (Fig. 3) may be due to technical limitations (qPCR after 30 minutes treatment at $4{ }^{\circ} \mathrm{C}$ to exclude the possibility of JX-594 replication step). Previous studies showed that extracellular acidity can affect vaccinia virus mobility ${ }^{16}$ and acidic solution can stimulate vaccinia virus entrance into host cell. ${ }^{7,13}$ In this study, aldosterone treatment stimulated JX-594 entrance possibly via intracellular alkalization. It was that aldosterone treatment could modify hydrogen conductance in variety of cells such as renal epithelia cells and smooth muscle cells. ${ }^{17,18}$ After pretreatment of aldosterone in this study, alkalinized intracellular $\mathrm{pH}$ was reversed by JX-594 treatment suggesting the possibility of use of extracelluar $\mathrm{H}^{+}$as a driving force on viral entrance.

It is possible that the $\mathrm{H}^{+}$gradient across the plasma membrane can affect vaccinia virus entrance depending on cell lines. Extracellular Hydrogen as a potential driving force for JX-594 entry is interesting because the tumor microenvironment is accompanied with chronic inflammation which provide acidic environment ${ }^{7,13}$ In addition to its inherent cancer tropism and genetic background vaccinia oncolytic virus, this may explain another factor for tumor specificity of JX-594.

In other aspect, vaccinia virus as a smallpox vaccination in human is being considered with the emergence of bioterrorism. ${ }^{19}$
However, post vaccination encephalitis was reported to be a serious safety concern for future vaccination ${ }^{20}$. Therefore, as is described in "Introduction", it is also interesting to note postvaccination encephalitis and cardiomyositis parallels with high level expression of MR in specific areas of the brain and cardiomyocytes. This study may provide the possibility of pharmacological modulation of fatal vaccinia virus amplification potentially minimizing postvaccination encephalitis and myopericardiomyositis. However, cytosolic $\mathrm{pH}$ involves many complexities which brought many study limitations and since our study did not include in vivo studies, further experiments are necessary to address the mechanisms involved. In conclusion, this study suggests potential pharmacological intervention in the replication of oncolytic vaccinia virus.

\section{Acknowledgements}

This work was supported by Pusan National University Research Grant, 2008.

\section{REFERENCES}

1. Heo J, Breitbach CJ, Moon A, Kim CW, Patt R, Kim MK, et al. Sequential therapy with JX-594, a targeted oncolytic poxvirus, followed by sorafenib in hepatocellular carcinoma: preclinical and clinical demonstration of combination efficacy. Mol Ther 2011;19:1170-1179.

2. Kim JS, Kim JH, Oh JY, Park BH, Yoon JH, Thorne SH, et al. Antitumoral efficacy of multiple injection of JX-594 (thymidine kinase (TK) deleted, human GM-CSF inserted Wyeth strain) via tail vein in $\mathrm{N}$-nitrosomorpholine (NNM) treated rats. [Abstract]. Mol Ther 2005; 11:S67.

3. Park BH, Hwang T, Liu TC, Sze DY, Kim JS, Kwon HC, et al. Use of a targeted oncolytic poxvirus, JX-594, in patients with refractory primary or metastatic liver cancer: a phase I trial. Lancet Oncol 2008;9:533-542.

4. Wu C, Orozco C, Boyer J, Leglise M, Goodale J, Batalov S, et al. BioGPS: an extensible and customizable portal for querying and organizing gene annotation resources. Genome Biol 2009;10:R130.

5. Mastrangelo MJ, Maguire HC Jr, Eisenlohr LC, Laughlin CE, Monken $\mathrm{CE}, \mathrm{McCue} \mathrm{PA}$, et al. Intratumoral recombinant GM-CSF-encoding virus as gene therapy in patients with cutaneous melanoma. Cancer Gene Ther 1999;6:409-422.

6. Thorne SH, Hwang TH, O'Gorman WE, Bartlett DL, Sei S, Kanji F, et al. Rational strain selection and engineering creates a broad-spectrum, systemically effective oncolytic poxvirus, JX-963. J Clin Invest 2007; 117:3350-3358.

7. Townsley AC, Weisberg AS, Wagenaar TR, Moss B. Vaccinia virus entry into cells via a low-pH-dependent endosomal pathway. J Virol 2006;80:8899-8908.

8. Seo JT, Steward MC, Larcombe-McDouall JB, Cook LJ, Case RM. Continuous fluorometric measurement of intracellular $\mathrm{pH}$ and $\mathrm{Ca} 2+$ in perfused salivary gland and pancreas. Pflugers Arch 1994;426:75-82.

9. Funder JW. Minireview: aldosterone and the cardiovascular system: genomic and nongenomic effects. Endocrinology 2006;147:5564-5567.

10. Finckenberg P, Mervaala E. Novel regulators and drug targets of cardiac 
hypertrophy. J Hypertens 2010;28(Suppl 1):S33-S38.

11. Ahokas RA, Warrington KJ, Gerling IC, Sun Y, Wodi LA, Herring PA, et al. Aldosteronism and peripheral blood mononuclear cell activation: a neuroendocrine-immune interface. Circ Res 2003;93:e124- e135.

12. Isobe A, Takeda T, Wakabayashi A, Tsuiji K, Li B, Sakata M, et al. Aldosterone stimulates the proliferation of uterine leiomyoma cells. Gynecol Endocrinol 2010;26:372-377.

13. Mercer J, Helenius A. Vaccinia virus uses macropinocytosis and apoptotic mimicry to enter host cells. Science 2008;320:531-535.

14. Gekle M, Golenhofen N, Oberleithner H, Silbernagl S. Rapid activation of $\mathrm{Na}+/ \mathrm{H}+$ exchange by aldosterone in renal epithelial cells requires $\mathrm{Ca} 2+$ and stimulation of a plasma membrane proton conductance. Proc Natl Acad Sci U S A 1996;93:10500-10504.

15. Oberleithner $\mathrm{H}$, Weigt $\mathrm{M}$, Westphale HJ, Wang W. Aldosterone activates $\mathrm{Na}+/ \mathrm{H}+$ exchange and raises cytoplasmic $\mathrm{pH}$ in target cells of the amphibian kidney. Proc Natl Acad Sci U S A 1987;84:1464-1468.

16. Taylor DH, Bosmann HB. Measurement of the electrokinetic properties of vaccinia and reovirus by laser-illuminated whole-particle microelectrophoresis. J Virol Methods 1981;2:251-260.

17. Wehling M, Bauer MM, Ulsenheimer A, Schneider M, Neylon CB, Christ M. Nongenomic effects of aldosterone on intracellular $\mathrm{pH}$ in vascular smooth muscle cells. Biochem Biophys Res Commun 1996; 223:181-186.

18. Vilella S, Guerra L, Helmle-Kolb C, Murer H. Aldosterone actions on basolateral $\mathrm{Na}+/ \mathrm{H}+$ exchange in Madin-Darby canine kidney cells. Pflugers Arch 1992;422:9-15.

19. Parrino J, Graham BS. Smallpox vaccines: Past, present, and future. J Allergy Clin Immunol 2006;118:1320-1326.

20. Miravalle A, Roos KL. Encephalitis complicating smallpox vaccination. Arch Neurol 2003;60:925-928. 